were compared to clinical and microbiological data (including time-to-positivity in liquid culture, TTP).

Results Of 79 patients, 44/79 (56\%) were male, median age 63 years [IQR 53;75]. CXR was performed median 5-days [IQR 2;28] from sample collection. Inter-rater CXR agreement was 92\%, kappa 0.57 [95\% CI: 0.52-0.63]. 58/79 (75\%) of subjects had an abnormal CXR [Table 1]: half had nodules and BWT, with 41\%, 23\% and 20\% bronchiectasis, consolidation and cavities respectively. Using symptoms present in $>1 / 3$ patients, only sputum and difficulty breathing were significantly associated with CXR score ( $\mathrm{p}=0.04 ; \mathrm{p}=0.01)$. M.avium and M.intracellulare were the most common NTM isolated [Table 1]. The highest abnormal median score for CXR was for M.xenopi $(6 / 30)$ followed by M.perigrinum (5/30), and M.abscessus (4.5/30). For M.kansasii there was significant correlation between CXR score and TTP $(\mathrm{r}=-0.82 ; \mathrm{p}=0.01)$ with cavitation being associated with a significantly lower TTP (4.5 vs 17.5 days; $\mathrm{p}=0.03$ ). CXR score did not predict whether or not a patient started treatment (OR 1.07; $\mathrm{p}=0.40)$.

Conclusion CXR abnormalities were present in $75 \%$ of patients in whom NTM was isolated. Unlike M.tuberculosis, the extent of radiographic changes correlated poorly with clinical symptoms or mycobacterial burden. Better simple, repeatable measures of NTM disease severity are required.

\begin{tabular}{|c|c|c|c|c|c|}
\hline NTM & $\mathrm{N}(\%)$ & $\begin{array}{l}\text { Abnormal } \\
\text { CXR } \\
\mathrm{N}(\%)\end{array}$ & Median CXR score & Median TTP (days) & \\
\hline M.avium & $24(30)$ & $20(25)$ & 3.5 & 8 & 5 \\
\hline M.intracelullare & $13(16)$ & $12(15)$ & 5 & 13.5 & 2 \\
\hline M.fortuitum & $12(15)$ & $7(9)$ & 4 & 9 & 0 \\
\hline M.kansasii & $8(10)$ & $4(5)$ & 2 & 6.5 & 3 \\
\hline M.chelonae & $5(6)$ & $3(4)$ & 3 & 9 & 0 \\
\hline M.peregrinum & $4(5)$ & $4(5)$ & 5 & 10 & 0 \\
\hline M.gordonae & $4(5)$ & $0(0)$ & 0 & 29 & 0 \\
\hline M.xenopi & $3(4)$ & $3(4)$ & 6 & 24 & 1 \\
\hline M.mucogencum & $2(3)$ & $2(3)$ & 2 & 12.5 & 0 \\
\hline M.abscessus & $2(3)$ & $1(1)$ & 4.5 & 5 & 0 \\
\hline M.palustre & $1(1)$ & $1(1)$ & 0 & 14 & 0 \\
\hline M.vulneris & $1(1)$ & 1 (1) & 4 & - & 1 \\
\hline
\end{tabular}

\section{M18 ENHANCED TRAINING FOR MEDICAL REGISTRARS LEARNING CHEST DRAINS AND THORACOCENTESIS. UNDERSTANDING TOTAL COMPETENCY LEVEL IN THE WORK FORCE}

AL Chapman, M Gingell, E Clissold. Waitemata District Health Board, Auckland, New Zealand

\subsection{6/thoraxjnl-2016-209333.460}

Performing a chest drain procedure with a safe technique is a core skill for a medical registrar and arguably the highest risk procedure they are expected to perform at junior level.

The competency level of a cohort of registrars who typically rotate every six months is often not known. Little regard is given
Level1 The trainee has insufficient knowledge and/or skill to perform the procedure

\section{Level 2 The trainee may perform the procedure under supervision}

\section{Level 3 The trainee may perform this procedure independently under exceptional circumstances}

\section{Level 4 The trainee may perform this procedure independently}

\section{Level 5 The trainee is suitable to supervise others in perform ing the procedure}

\section{Not assessed}

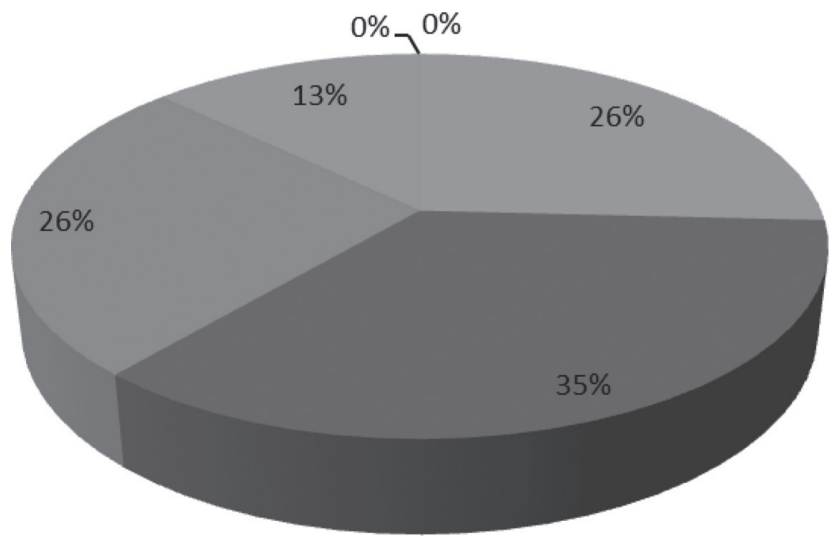

Abstract M18 Figure 2 Seldinger competence

to this when drawing up on call rotas and training needs are often missed. In this initiative we aimed to address these failings. Methods By way of induction for the general medical registrars beginning a six month post in our hospitals we arranged a short verbal introduction to the group about local processes of how chest procedures within our hospital are performed eg. location of equipment in centralised packs, standardised equipment lists, check lists and use of bedside US and patient information/ consent.

Over the first two weeks of the run we arranged "one on one" teaching with a Consultant chest physician who taught each registrar according to their needs on realistic mannequins. Three techniques taught: thoracocentesis with an $8 \mathrm{~F}$ drainage pack; a small bore $12 \mathrm{~F}$ seldinger drain and a surgical $28 \mathrm{~F}$ drain drain according to need. Teaching of 32 registrars was completed within a two week time frame in the space of four $\mathrm{PA} /$ clinic sessions. Competency was judged at the end of the session and electronic DOPS and feedback from the registrars was obtained. We encouraged further discussion with educational supervisors with regard to ongoing training requirements for individuals. 
Results and evaluation Attendance was 31/32. 59\% had 12 months or less experience as a registrar. $66 \%$ were able to perform thoracocentesis and 63\% seldinger drain independently. Most others were competent enough to perform with a colleague supervising. Additionally $38 \%$ and $25 \%$ of registrars were deemed to be able to teach other thoracocentesis and seldinger drains respectively.

Learner feedback was extremely positive and self-rated confidence and safety improved from 5-7.6 and 5.2 to 7.7 out of 10 respectively. Responses highly valued the fact this was delivered by a Consultant and identified a need for further bedside chest US training.

\section{M19 PATIENT PERSPECTIVES OF AN AMBULATORY PLEURAL SERVICE}

N Devani, U Gupta, R Kaiser, RH Johns. Barking, Havering and Redbrige University Hospitals NHS Trust, Essex, UK

\subsection{6/thoraxjnl-2016-209333.461}

Background and objectives Barking, Havering and Redbridge University Trust (BHRUT) serves a population of 750,000 patients with a large burden of pleural disease. Patients admitted and requiring pleural drainage usually results in a long length of stay of around 7-10 days. Outpatient ambulatory management of undiagnosed and known malignant pleural effusions is increasing nationally in the UK through development of pleural clinics. Previous reports have demonstrated these to be financially efficacious and avoid hospitalisation. We sought to demonstrate that they are also well received and favoured by patients.

Methods In December 2015, an outpatient weekly pleural aspiration service was established receiving referrals directly from respiratory outpatients, $A \& E$ and acute medicine. We prospectively audited patients attending this service between December 2015 and June 2016. Patients were asked to complete an in-depth questionnaire to assess their experience on the day, any procedural discomfort and attitudes toward such an outpatient service. Results 81 patients attended our service over this period. Median age was 74 (range 30-92), 40\% female. 58 patients returned a completed questionnaire. $86 \%$ of patients were seen within a week of referral with the rest waiting less than 2 weeks. The majority $(74 \%, n=43)$ of patients did not notice any deterioration in their symptoms during this wait. Median pain score was 3 (range $1-10) .78 \%(n=45)$ of patients felt they could continue with their normal activities post procedure. Only 2 patients would have preferred to undergo the procedure as an inpatient citing frailty as the reason. $98 \%(n=57)$ of patients felt that an outpatient pleural service was a good idea. 78\% $(n=45)$ rated the service as excellent, $17 \%(\mathrm{n}=10)$ as 'above average' and only $5 \%(\mathrm{n}=3)$ as 'average.'

Conclusions Outpatient management of pleural effusions is favoured by patients with most rating our service as excellent. Patients are seen promptly with the majority reporting no deterioration in their symptoms during the wait. The procedure is well tolerated and allows patients to continue with their normal daily activities. In addition to important financial benefits of reducing hospital bed-days in patients with pleural effusions, our newly established service has been shown to be beneficial in promoting a positive patient experience. HOSPITAL - 1 YEAR RETROSPECTIVE REVIEW

A Fawzi, N Maddekar, S Khan, S Bikmalla, M Iqbal, AKA Abi Musa Asa'ari, T Cusay, A Austin, C Moore, M Haris. Royal Stoke University Hospital, Stoke-on-Trent, UK

10.1136/thoraxjnl-2016-209333.462

Introduction Over the last decade, there has been a paradigm shift in the management of both pleural effusions and pneumothoraces. Specialty pleural day-case services have been established offering one-stop clinical assessment, imaging and intervention, with a view to early diagnosis, improved patient care and admission avoidance. We wished to evaluate the pleural service at a large university teaching Hospital.

Methods 650 consecutive outpatient episodes presenting to the pleural day-case clinic between January 2015 and January 2016 were reviewed. Patient characteristics, source of referral, diagnosis, interventions and outcomes were evaluated.

Results Total number of patient episodes: 650. 264 new outpatients, 322 outpatient follow-ups, 64 pleural in-reach. Male 403 (62\%), Female 247 (38\%). Referral Source: GP 151 (58\%), acute medicine unit/A\&E/other specialties 103 (38\%) and external referrals 10 (4\%).

Pleural Interventions: 27 diagnostic aspirations, 113 therapeutic aspirations, 27 chest drain insertions, 37 indwelling pleural catheters.

Indications for referrals: Pneumothorax: 24, 27 parapneumonic/Complicated effusions, 57 known malignancy, 150 unknown effusions, 8 others.

Average length of stay (based on the BTS Pleural audit 2014) ${ }^{1}$ was 8 days. Admission avoidance: 264 patients $\times 8$ days $=2,112$ bed days saved in 48 weeks. Early discharge assuming 4 days (66 patients $\times 4$ days $)=264$ bed days saved. Total bed days saved $=$ 2376 in 48 weeks - Equivalent of 7 bed days per patient.

Conclusions A dedicated pleural service has resulted in improvement in both patient outcomes and experience. The number of unnecessary pleural procedures has reduced. Complicated cases are discussed in Pleural MDT meetings. A recent patient feedback survey conducted over 2 months has shown a highly favourable patient experience of the service itself. The pleural service has allowed the department to recruit to several appropriate NIHR trials. The number of bed days saved is significant, raising the question as to whether a nationwide adoption of pleural services in the majority of trusts, would take some of the strain off of an overburdened NHS.

\section{REFERENCE}

1 Hooper CE, Welham SA, Maskell NA. Pleural procedures and patient safety: a national BTS audit of practice. Thorax 2013;70(2):189-191.

\section{M21 PLEURAL EFFUSION SIZE - A RETROSPECTIVE COMPARISON OF COMPUTED TOMOGRAPHY AND ULTRASOUND REPORTING}

MG Aldik, VM Raju, C Daneshvar. Plymouth Hospitals NHS Trust, Plymouth, UK

\subsection{6/thoraxjnl-2016-209333.463}

Background Pleural effusion size may determine patient management and is routinely reported using thoracic computed tomography (CT) and ultrasound scans. We aimed to compare agreement between these two modalities.

Methods Between August 2015 and January 2016, patients referred through the pleural service with pleural effusions were 